\title{
Implementation of reduced form mechanisms: a simple approach and a new characterization
}

\author{
Sergiu Hart • Philip J. Reny
}

Received: 25 July 2014 / Accepted: 4 August 2014 / Published online: 22 August 2014

(C) Society for the Advancement of Economic Theory 2014

\begin{abstract}
We provide a new characterization of implementability of reduced form mechanisms in terms of straightforward second-order stochastic dominance. In addition, we present a simple proof of Matthews (1984) conjecture, proved by Border (1991), on implementability.
\end{abstract}

Keywords Mechanism design $\cdot$ Auctions $\cdot$ Reduced form mechanism $\cdot$ Stochastic dominance $\cdot$ Implementability $\cdot$ Interim probabilities

JEL Classification $\quad \mathrm{C} 6 \cdot \mathrm{C} 7 \cdot \mathrm{D} 4$

\section{Introduction}

In mechanism design contexts, such as those with type-dependent outside options, it is sometimes more natural and convenient to optimize over reduced form mechanisms-

Previous versions: August 2010, December 2011, May 2013. Research partially supported by the National Science Foundation (SES-0922535) and the European Research Council (FP7-249159). The authors thank Benny Moldovanu, Yosi Rinott, Marco Scarsini, Ricky Vohra, and Benjy Weiss, for useful discussions and suggestions.

S. Hart $(\bowtie)$

Department of Economics, Institute of Mathematics, and Center for the Study of Rationality, The Hebrew University of Jerusalem, Jerusalem, Israel

e-mail: hart@huji.ac.il

URL: http://www.ma.huji.ac.il/hart

P. J. Reny

Department of Economics, University of Chicago, Chicago, USA

e-mail: preny@uchicago.edu

URL: http://home.uchicago.edu/ preny 
i.e., interim probability assignments and cost functions - rather than the underlying mechanisms themselves. ${ }^{1}$ Reduced form mechanisms also play a useful role in the literature on the equivalence between Bayesian and dominant strategy implementation. ${ }^{2}$ In contexts such as these, it is important to know when a reduced form mechanism can actually be implemented. Maskin and Riley (1984) posed and studied this question, and obtained a partial solution. Matthews (1984) made further progress and in addition conjectured that an intuitive inequality constraint is necessary and sufficient for implementability. Border (1991) finally solved the problem by proving Matthews' conjecture.

The purpose of the present note is twofold. First, we provide an alternative characterization of implementability in terms of straightforward second-order stochastic dominance, and second, we offer a simple and direct proof of Matthews' conjecture. Reducing the problem to a well-studied concept - in this case stochastic dominanceputs at our disposal a wide range of tools and insights; see for instance Remarks (b)-(d) below.

Our work, like all of the work mentioned above, focuses on the classic mechanism design setting in which there is a single indivisible object to be allocated to one of $n$ ex-ante symmetric agents, each of whose private information, which may be quite general, is drawn independently from a common distribution. ${ }^{3}$

\section{Results}

The underlying probability space (the "type space") is ${ }^{4}(T, \mathcal{T}, \lambda)$. The number of agents is $n$. Because the implementability question relates only to a mechanism's probability assignment function, we define a mechanism here in these terms only. ${ }^{5}$ Thus, a mechanism consists of $n$ functions $q_{1}, q_{2}, \ldots, q_{n}$, with $q_{i}: T^{n} \rightarrow[0,1]$ for each $i=1,2, \ldots, n$, such that $\sum_{i=1}^{n} q_{i}\left(t_{1}, t_{2}, \ldots, t_{n}\right) \leq 1$ for every $t_{1}, t_{2}, \ldots, t_{n}$ in $T$; here $q_{i}\left(t_{1}, t_{2}, \ldots, t_{n}\right)$ is the probability that agent $i$ gets the object when the reported types are $t_{1}, t_{2}, \ldots, t_{n}$. A mechanism is symmetric if $q_{i}\left(t_{\pi(1)}, t_{\pi(2)}, \ldots, t_{\pi(n)}\right)=$ $q_{\pi(i)}\left(t_{1}, t_{2}, \ldots, t_{n}\right)$ for every permutation $\pi$ of $\{1,2, \ldots, n\}$ and every agent $i=$ $1,2, \ldots, n$; i.e., the "names" $(1,2, \ldots, n)$ of the agents do not matter. A symmetric mechanism is thus given by a function $q \equiv q_{1}: T^{n} \rightarrow[0,1]$ such that $q\left(t_{1}, t_{2}, \ldots, t_{n}\right)$ is invariant to permutations of $\left(t_{2}, \ldots, t_{n}\right)$ and $\sum_{i} q_{i} \leq 1$, where $q_{i}\left(t_{1}, t_{2}, \ldots, t_{n}\right):=q\left(t_{i}, t_{2}, \ldots, t_{i-1}, t_{1}, t_{i+1}, \ldots, t_{n}\right)$ (i.e., interchange the first and the $i$ th coordinates); $q\left(t_{1}, t_{2}, \ldots, t_{n}\right)$ is the probability that an agent whose type is $t_{1}$ gets the object when the other agents are of types $t_{2}, \ldots, t_{n}$.

\footnotetext{
1 See, e.g., Mierendorff (2009); in the algorithmic game-theoretic literature, see Cai et al. (2012).

2 See, e.g., Manelli and Vincent (2010) and Gershkov et al. (2013).

3 For the case of asymmetric agents with finite-type spaces, see Border (2007) and Che et al. (2013); see also Mierendorff (2011).

4 There are no requirements on the probability space. All functions will be assumed measurable, and all statements to hold almost surely.

5 Thus the cost function as well as additional constraints (such as participation constraints), which are not important for our purposes, are left unspecified.
} 
A reduced form is a mapping $Q: T \rightarrow[0,1]$. A reduced form $Q$ is implementable 6 if there exists a symmetric mechanism given by $q: T^{n} \rightarrow[0,1]$ such that $Q(t)=$ $\int_{T^{n-1}} q\left(t, t_{2}, \ldots, t_{n}\right) \mathrm{d} \lambda\left(t_{2}\right) \cdots \mathrm{d} \lambda\left(t_{n}\right)$ for all $t \in T$; i.e., $Q(t)$ is the overall probability that an agent of type $t$ gets the object. In this case we say that $Q$ is the reduced form of $q$, or that $q$ generates $Q$. Our concern is whether a given reduced form $Q$ is implementable. ${ }^{7}$

As will be shown, an important special case consists of the type space $\left([0,1], \mathcal{B}, \lambda^{*}\right)$, with $\lambda^{*}$ the Lebesgue measure, together with the "efficient" mechanism $q^{*}\left(t_{1}, t_{2}, \ldots, t_{n}\right)$ $=1$ if $t_{1}>\max \left\{t_{2}, \ldots, t_{n}\right\}$, and $q^{*}\left(t_{1}, t_{2}, \ldots, t_{n}\right)=0$ otherwise. That is, the agents' types are each uniformly distributed on $[0,1]$ and the mechanism gives the object to the agent whose type is highest. Its reduced form is easily seen to be $Q^{*}(t)=t^{n-1}$ for all $t \in[0,1]$ (when $n=2$, the distribution of $Q^{*}$ is uniform on $[0,1]$ ).

Theorem 1 The following conditions on $Q: T \rightarrow[0,1]$ are equivalent:

(i) $Q$ is implementable; i.e., it is the reduced form of a symmetric mechanism.

(ii) $Q$ satisfies, for every ${ }^{8} \alpha \in[0,1]$,

$$
\int_{[Q>\alpha]} Q(t) \mathrm{d} \lambda(t) \leq \frac{1}{n}-\frac{1}{n}(\lambda[Q \leq \alpha])^{n} .
$$

(iii) $-Q$ second-order stochastically dominates $-Q^{*}$.

\section{Remarks.}

(a) Condition (ii) is due to Matthews (1984); ${ }^{9}$ Border (1991) shows the equivalence of (i) and (ii). ${ }^{10}$ Condition (iii) is new.

(b) Condition (iii) means that for every increasing and concave function $u$ : $[-1,0] \rightarrow \mathbb{R}$ we have ${ }^{11} \mathbf{E}[u(-Q)] \geq \mathbf{E}\left[u\left(-Q^{*}\right)\right]$; equivalently, for every

\footnotetext{
6 We follow the literature in referring to this property as "implementability"; it is in fact only a feasibility requirement (and has nothing to do with incentive constraints and game-theoretic notions of implementation).
}

7 The symmetry requirement here, though natural, is somewhat more demanding than that in Maskin and Riley (1984) and Border (1991), who do not require the invariance of $q\left(t_{1}, t_{2}, \ldots, t_{n}\right)$ with respect to permutations of $\left(t_{2}, \ldots, t_{n}\right)$. But this strengthening has no effect on the implementability of a reduced form $Q$, since, for any mechanism $q$, averaging all its $n$ ! permutations $\pi$ yields a mechanism $\tilde{q}$ that is symmetric in our sense and has the same reduced form (specifically, $\tilde{q}_{i}\left(t_{1}, \ldots, t_{n}\right):=$ $\left.(1 / n !) \sum_{\pi} q_{\pi^{-1}(i)}\left(t_{\pi(1)}, t_{\pi(2)}, \ldots, t_{\pi(n)}\right)\right)$.

${ }^{8}[Q<\alpha]$ is short for $\{t \in T: Q(t)<\alpha\}$; similarly for the other events.

9 The fact that it suffices to consider the inequality (1) only on sets of the form $[Q>\alpha]$, rather than on all measurable sets as in Matthews (1984), is immediate (see Proposition 3.2 of Border 1991).

10 When there are only two agents (i.e., $n=2$ ), the implementability of $Q$ can be restated as the existence of a joint distribution with density that is bounded (by $1 / \mathbf{E}[Q]$ ) and has given marginals (namely, the distributions of appropriate rescalings of $Q$ and $1-Q$ ). See Lorenz (1949), Kellerer (1961), and Strassen (1965) for a general solution to such problems, which can then be proved to yield Matthews' condition (ii). Gale (1957) and Ryser (1957) deal with the related problem of the existence of matrices with entries 0 or 1 that have given row and column sums; interestingly, the resulting condition can be stated in terms of majorization, the discrete version of second-order stochastic dominance. While this result is restricted to only two agents, it may nevertheless be interesting to explore the connection further. We thank Benny Moldovanu for referring us to this literature.

$11 \mathbf{E}$ denotes expectation (with respect to the appropriate probability measure: $\lambda$ for $Q$, and $\lambda^{*}$ for $Q^{*}$ ). 
increasing and convex function $v:[0,1] \rightarrow \mathbb{R}($ take $u(x)=-v(-x)$ ), we have $\mathbf{E}[v(Q)] \leq \mathbf{E}\left[v\left(Q^{*}\right)\right]$, or

$$
\int_{T} v(Q(t)) \mathrm{d} \lambda(t) \leq \int_{T^{*}} v\left(Q^{*}(t)\right) \mathrm{d} \lambda^{*}(t)=\int_{0}^{1} v\left(t^{n-1}\right) \mathrm{d} t .
$$

In terms of distributions, this amounts to $Q^{*}$ being obtained from $Q$ by increasing values (pointwise) and applying mean-preserving spreads (see Hadar and Russell 1969; Hanoch and Levy 1969; Rothschild and Stiglitz 1970; and the book of Shaked and Shanthikumar 2010).

Equivalently, there exists a probability space $(\Omega, \mathcal{F}, \mathbf{P})$ and two random variables $X$ and $Y$ defined on it, such that $X$ and $Q$ have the same distribution, $Y$ and $Q^{*}$ have the same distribution, and ${ }^{12} \mathbf{E}[Y \mid X] \geq X$; this is a standard construct, which translates stochastic dominance into pointwise (a.s.) dominance, known as coupling (see Strassen 1965, Theorem 9; Machina and Pratt 1997, Theorem 3'; Shaked and Shanthikumar 2010, Theorem 4.A.5).

(c) An immediate consequence of condition (iii) (see (2) above) is that, for each increasing and convex function $v$, the maximum of $\mathbf{E}[v(Q)]$ over all implementable $Q$ is attained at $Q^{*}$ and equals $\int_{0}^{1} v\left(t^{n-1}\right) \mathrm{d} t$.

(d) When $Q$ takes only finitely many values (which happens in particular when $T$ is a finite set), say the values $r_{1}<r_{2}<\cdots<r_{m}$ with probabilities $\rho_{1}, \rho_{2}, \ldots, \rho_{m}>0$, respectively (so $\sum_{k=1}^{m} \rho_{k}=1$ ), we may replace in condition (iii) the function $Q^{*}$ with another function $Q^{\#}$ that takes the $m$ values $s_{1}<s_{2}<\cdots<s_{m}$ with probabilities $\rho_{1}, \rho_{2}, \ldots, \rho_{m}$, respectively, where $s_{k}:=\left(n \rho_{k}\right)^{-1}\left[\left(\sum_{\ell=1}^{k} \rho_{\ell}\right)^{n}-\left(\sum_{\ell=1}^{k-1} \rho_{\ell}\right)^{n}\right]$ for $1 \leq k \leq m$. The resulting condition is

$$
\left(\text { iii }^{\#}\right)-Q \text { second-order stochastically dominates }-Q^{\#} \text {, }
$$

which can be expressed as

$$
\sum_{\ell=k}^{m} \rho_{\ell} r_{\ell} \leq \sum_{\ell=k}^{m} \rho_{\ell} s_{\ell}=\frac{1}{n}-\frac{1}{n}\left(1-\sum_{\ell=k}^{m} \rho_{\ell}\right)^{n} \quad \text { for every } 1 \leq k \leq m
$$

This inequality is the "weighted majorization" of the sequence $\left(r_{k}\right)$ by the sequence $\left(s_{k}\right)$, relative to the weights ${ }^{13}\left(\rho_{k}\right)$. To see that (iii $\left.{ }^{\#}\right)$ implies (iii), use the fact that $Q^{\#}$ has the same distribution as the conditional expectation of $Q^{*}$ when $[0,1]$ is partitioned into $m$ intervals of lengths $\rho_{1}, \rho_{2}, \ldots, \rho_{m}$ (the partition points being $\rho_{1}, \rho_{1}+\rho_{2}, \ldots, \rho_{1}$

12 I.e., $\mathbf{P}[X \leq \alpha]=\lambda[Q \leq \alpha]$ and $\mathbf{P}[Y \leq \alpha]=\lambda^{*}\left[Q^{*} \leq \alpha\right]=\alpha^{1 /(n-1)}$ for every $\alpha \in[0,1]$. The change from $X$ to $Y$ can be understood as increasing values pointwise (from $X$ to $\mathbf{E}[Y \mid X]$ ) and applying mean-preserving spreads (from $\mathbf{E}[Y \mid X]$ to $Y$ ).

13 When all the weights are equal, i.e., $\rho_{k}=1 / m$ for all $k$, this is the classical majorization-see Hardy et al. (1952, Theorem 46); their proof of Theorem 46 easily generalizes to arbitrary weights. An alternative simple proof for the equivalence of (iiï ${ }^{\#}$ ) and (3) is obtained using Theorem 3.A.5 in Shaked and Shanthikumar (2010). 
$\left.+\cdots+\rho_{m-1}=1-\rho_{m}\right)$, and so $-Q^{\#}$ second-order stochastically dominates ${ }^{14}-Q^{*}$. Conversely, taking $\alpha$ converging from the left to $r_{k}$ in (1) gives (3), and so (ii) implies (iii ${ }^{\#}$ ).

\section{Proof of Theorem 1. ${ }^{15}$}

- (i) implies (ii). As in Matthews (1984) and Border (1991, Lemma 5.1), using symmetry and then $\sum_{i} q_{i} \leq 1$ yields for any measurable $A \subset T$, in particular for ${ }^{16}$ $A=[Q>\alpha]$,

$$
\begin{aligned}
n \mathbf{E}\left[Q(t) \mathbf{1}_{[t \in A]}\right] & =\mathbf{E}\left[\sum_{i=1}^{n} Q\left(t_{i}\right) \mathbf{1}_{\left[t_{i} \in A\right]}\right]=\mathbf{E}\left[\sum_{i=1}^{n} q_{i}\left(t_{1}, \ldots, t_{n}\right) \mathbf{1}_{\left[t_{i} \in A\right]}\right] \\
& \leq \mathbf{E}\left[\sum_{i=1}^{n} q_{i}\left(t_{1}, \ldots, t_{n}\right) \mathbf{1}_{\cup_{j}\left[t_{j} \in A\right]}\right] \leq \mathbf{E}\left[\mathbf{1}_{\cup_{j}\left[t_{j} \in A\right]}\right]=1-(\lambda[T \backslash A])^{n} .
\end{aligned}
$$

- (ii) implies (iii). Put $p:=\lambda[Q \leq \alpha]$; we have ${ }^{17}$

$$
\begin{aligned}
\int_{T}[Q(t)-\alpha]_{+} \mathrm{d} \lambda(t) & =\int_{[Q>\alpha]} Q(t) \mathrm{d} \lambda(t)-\alpha(1-p) \leq \frac{1}{n}-\frac{1}{n} p^{n}-\alpha+\alpha p \\
& \leq \frac{1}{n}-\alpha+\frac{n-1}{n} \alpha^{\frac{n}{n-1}}=\int_{0}^{1}\left[t^{n-1}-\alpha\right]_{+} \mathrm{d} t \\
& =\int_{T^{*}}\left[Q^{*}(t)-\alpha\right]_{+} \mathrm{d} \lambda^{*}(t)
\end{aligned}
$$

where the first inequality is (1), and the second is equivalent to $\alpha p \leq(1 / n) p^{n}+$ $((n-1) / n) \alpha^{n /(n-1)}$, which follows easily by concavity after taking the log of both sides. ${ }^{18}$ Hence $\int_{T}[Q-\alpha]_{+} \mathrm{d} \lambda \leq \int_{T^{*}}\left[Q^{*}-\alpha\right]_{+} \mathrm{d} \lambda^{*}$ for all $\alpha \in[0,1]$, which is equivalent to (2) since every increasing convex function $v$ (with $v(0)=0$, which does not affect (2)) lies in the closed convex cone generated by the functions $\phi_{\alpha}(x):=[x-\alpha]_{+}$for all $\alpha \in[0,1]{ }^{19,20}$

14 Since $\mathbb{E}\left[Q^{*}\right]=\mathbb{E}\left[Q^{\#}\right]$ this is the same as $Q^{\#}$ second-order stochastically dominating $Q^{*}$. Also, stochastic dominance is a transitive relation.

15 Showing that conditions (ii) and (iii) are each necessary for the implementability of $Q$ is quite straightforward; the difficulty lies in proving that these conditions are sufficient (cf. "(iii) implies (i)").

$16 \mathbf{1}_{W}$ denotes the indicator of the event $W$.

$17[x]_{+}:=\max \{x, 0\}$.

18 Alternatively, the second inequality follows by maximizing the left-hand side over $p$ (this is an instance of the classical Young inequality).

19 While for each $\alpha$ the inequality in (4), which can be written as $\int_{[Q>\alpha]} Q(t) \mathrm{d} \lambda(t) \leq 1 / n+((n-$ 1)/n) $\alpha^{n /(n-1)}-\alpha \lambda[Q \leq \alpha]$, is strictly weaker than inequality (1), our result implies that "(4) for all $\alpha$ " is equivalent to "(1) for all $\alpha$ " (this equivalence can also be proved quite directly). Of course, our purpose here is to provide a simple and self-contained proof of the equivalence of (i)-(iii).

20 In the special case of a finite type space $T$, condition (ii) generates a polymatroid (i.e., polytopes whose efficient frontier is the core of a convex game); see Vohra (2011, Section 6.2). In this case, an indirect proof of "(ii) implies (iii)" can be obtained by, first, characterizing the extreme points of the polymatroid in terms 
- (iii) implies (i). Assume that $-Q$ second-order stochastically dominates $-Q^{*}$. Applying coupling (see Remark (b)) yields a probability space and two random variables $X$ and $Y$ defined on it, such that $X$ and $Q$ have the same distribution, $Y$ and $Q^{*}$ have the same distribution, and $\mathbf{E}[Y \mid X] \geq X$.

Let $\left(X_{i}, Y_{i}\right)$, for $i=1,2, \ldots, n$, be $n$ independent pairs of random variables, all identically distributed and with the same distribution as the pair $(X, Y)$. For each $\left(t_{1}, t_{2}, \ldots, t_{n}\right) \in T^{n}$ define the event

$$
A\left(t_{1}, t_{2}, \ldots, t_{n}\right):=\left[X_{1}=Q\left(t_{1}\right), X_{2}=Q\left(t_{2}\right), \ldots, X_{n}=Q\left(t_{n}\right)\right]
$$

and put $^{21}$

$$
\tilde{q}\left(t_{1}, t_{2}, \ldots, t_{n}\right):=\mathbf{P}\left[Y_{1}>\max _{j \neq 1} Y_{j} \mid A\left(t_{1}, t_{2}, \ldots, t_{n}\right)\right] .
$$

Then $^{22} \sum_{i} \tilde{q}_{i}\left(t_{1}, \ldots, t_{n}\right)=\sum_{i} \mathbf{P}\left[Y_{i}>\max _{j \neq i} Y_{j} \mid A\left(t_{1}, \ldots, t_{n}\right)\right] \leq 1$ (these $n$ events are disjoint), and so $\tilde{q}: T^{n} \rightarrow[0,1]$ yields a symmetric mechanism. Moreover, integrating over $\left(t_{2}, \ldots, t_{n}\right) \in T^{n-1}$ (recall that $\left(Q\left(t_{2}\right), \ldots, Q\left(t_{n}\right)\right)$ and $\left(X_{2}, \ldots, X_{n}\right)$ have the same distribution) gives the reduced form $\tilde{Q}$ of $\tilde{q}$ :

$$
\tilde{Q}\left(t_{1}\right)=\mathbf{P}\left[Y_{1}>\max _{j \neq 1} Y_{j} \mid X_{1}=Q\left(t_{1}\right)\right] \text {. }
$$

Now $\mathbf{P}\left[Y_{j} \leq y\right]=\lambda^{*}\left[Q^{*} \leq y\right]=y^{1 /(n-1)}$ for every $y \in[0,1]$ (since $Y_{j}$ has the same distribution as $Q^{*}$, which implies that $\mathbf{P}\left[\max _{j \neq 1} Y_{j} \leq y \mid X_{1}\right]=$ $\prod_{j=2}^{n} \mathbf{P}\left[Y_{j} \leq y\right]=\left(y^{1 /(n-1)}\right)^{n-1}=y$ (we have used here the independence over $j)$. Thus $\max _{j \neq 1} Y_{j}$ is uniformly distributed on $[0,1]$, and moreover independent of $X_{1}$; hence (5) yields

$$
\tilde{Q}\left(t_{1}\right)=\int_{0}^{1} \mathbf{P}\left[Y_{1}>y \mid X_{1}=Q\left(t_{1}\right)\right] \mathrm{d} y=\mathbf{E}\left[Y_{1} \mid X_{1}=Q\left(t_{1}\right)\right] \geq Q\left(t_{1}\right)
$$

(recall that $\mathbf{E}\left[Y_{1} \mid X_{1}\right] \geq X_{1}$ ). It only remains to rescale: put

$$
q\left(t_{1}, \ldots, t_{n}\right):=\tilde{q}\left(t_{1}, \ldots, t_{n}\right) \frac{Q\left(t_{1}\right)}{\tilde{Q}\left(t_{1}\right)}
$$

(where we take $0 \cdot 0 / 0$ as 0 ); then $q$ yields a symmetric mechanism ( $\operatorname{since} q \leq \tilde{q}$ ), and its reduced form is precisely the given $Q$.

\footnotetext{
Footnote 20 continued

of "priority rules," and second, using this characterization to show that these points satisfy condition (iii). We thank Ricky Vohra for pointing this out.

21 We write $\mathbf{P}$ for the probability measure on the space on which all $X_{i}$ and $Y_{i}$ are defined.

22 Recall that $\tilde{q}_{i}$ is obtained from $\tilde{q}_{1} \equiv \tilde{q}$ by interchanging $t_{i}$ and $t_{1}$.
} 
Finally, consider symmetric mechanisms that are maximal, in the sense that $\sum_{i=1}^{n} q_{i}=1$. If $Q$ is the reduced form, then $\mathbf{E}\left[\sum_{i} q_{i}\right]=n \mathbf{E}[Q]$. It follows that an implementable reduced form $Q$ is the reduced form of a maximal mechanism if and only if $\mathbf{E}[Q]=1 / n$; in this case we will also call $Q$ maximal. Clearly, for any implementable $Q$ there is a maximal implementable $\tilde{Q}$ with $\tilde{Q}(t) \geq Q(t)$ for $\operatorname{all}^{23} t$. We have:

Corollary 2 The following conditions on $Q: T \rightarrow[0,1]$ with $\mathbf{E}[Q]=1 / n$ are equivalent:

(i-Max) $Q$ is the reduced form of a maximal symmetric mechanism. (iii-Max) $Q$ second-order stochastically dominates $Q^{*}$.

Proof $\mathbf{E}[Q]=1 / n$ implies that $\mathbf{E}[Q]=\mathbf{E}\left[Q^{*}\right]$ (since $Q^{*}$ is maximal), and in this case condition (iii) is equivalent to (iii-Max): indeed, for the coupled random variables $X$ and $Y$ of Remark (b), when $\mathbf{E}[X]=\mathbf{E}[Y]$ the conditions $\mathbf{E}[Y \mid X] \geq X$, $\mathbf{E}[Y \mid X]=X$, and $\mathbf{E}[Y \mid X] \leq X$, are all equivalent. ${ }^{24}$

Thus, $Q^{*}$ is obtained from an implementable maximal $Q$ by mean-preserving spreads; that is, $Q^{*}$ has the same distribution as $Q+Z$ for some "noise" $Z$ that is uncorrelated with $Q$ (i.e., $\mathbf{E}[Z \mid Q]=0$ ).

\section{References}

Border, K.C.: Implementation of reduced form auctions: a geometric approach. Econometrica 59, 11751187 (1991)

Border, K.C.: Reduced form auctions revisited. Econ. Theory 31, 167-181 (2007)

Cai, Y., Daskalakis, C., Weinberg, S. M.: An algorithmic characterization of multi-dimensional mechanisms. In: STOC 2012: Proceedings of the Forty-Fourth Annual ACM Symposium on Theory of Computing, pp. 459-478 (2012)

Che, Y.-K., Kim, J., Mierendorff, K.: Generalized reduced-form auctions: a network-flow approach. Econometrica 81, 2487-2520 (2013)

Gale, D.: A theorem on flows in networks. Pac. J. Math. 7, 1073-1082 (1957)

Gershkov, A., Goeree, J.K., Kushnir, A., Moldovanu, B., Shi, A.: On the equivalence of Bayesian and dominant strategy implementation. Econometrica 81, 197-220 (2013)

Hadar, J., Russell, W.: Rules for ordering uncertain prospects. Am. Econ. Rev. 59, 25-34 (1969)

Hanoch, G., Levy, H.: The efficiency analysis of choices involving risk. Rev. Econ. Stud. 36, 335-346 (1969)

Hardy, G., Littlewood, J. E., Pólya, G.: Inequalities, Second Edition, Cambridge University Press (1952)

Kellerer, H.G.: Funktionen auf Produkträumen mit vorgegebenen Marginal-Funktionen. Math. Ann. 144, 323-344 (1961)

Lorenz, G.G.: A problem of plane measure. Am. J. Math. 71, 417-426 (1949)

Machina, M., Pratt, J.: Increasing risk: some direct constructions. J. Risk Uncertain. 14, 103-127 (1997)

Manelli, A., Vincent, D.: Bayesian and dominant strategy implementation in the independent, private values model. Econometrica 78, 1905-1939 (2010)

Maskin, E.S., Riley, J.: Optimal auctions with risk-averse buyers. Econometrica 52, 1473-1518 (1984)

Matthews, S.A.: On the implementability of reduced form auctions. Econometrica 52, 1519-1522 (1984)

\footnotetext{
23 If $Q$ is the reduced form of $q$, then let $\tilde{Q}$ be the reduced form of $\tilde{q}$, which is defined by $\tilde{q}:=q / \sum_{i} q_{i}$ (when $q_{i}=0$ for all $i$ take $\tilde{q}:=1 / n$ ).

24 Informally: when the expectations are equal one cannot increase the values, and only mean-preserving spreads can be used.
} 
Mierendorff, K.: Optimal dynamic mechanism design with deadlines. University of Bonn (2009). Mierendorff, K.: Asymmetric reduced form auctions. Econ. Lett. 110, 41-44 (2011)

Ryser, H.J.: Combinatorial properties of matrices of zeros and ones. Can. J. Math. 9, 371-377 (1957)

Rothschild, M., Stiglitz, J.: Increasing risk: I. A definition. J. Econ. Theory 2, 225-243 (1970)

Shaked, M., Shanthikumar, J.G.: Stochastic Orders, Springer (2010).

Strassen, V.: The existence of probability measures with given marginals. Ann. Math. Stat. 36, 423-439 (1965)

Vohra, R.V.: Mechanism design: a linear programming approach. Cambridge University Press (2011). 